\title{
Approach to percutaneous nephrolithotomy. Comparison of the procedure in a one-shot versus the sequential with metal dilatators
}

\author{
Ismael Sedano-Portillo', Gastón Ochoa-León ${ }^{1}$, Clotilde Fuentes-Orozco², Leire Irusteta-Jiménez², \\ Luis Rodrigo Michel-Espinoza², Marcela Salazar-Parra², Lizbeth Cuesta-Márquez ${ }^{2}$ and \\ Alejandro González-Ojeda² \\ ${ }^{1}$ Department of Urology; ${ }^{2}$ Biomedical Research Unit 02, Specialty Hospital. National Medical Center of the West, Mexican Institute of Social Security, \\ Guadalajara, Jal., Mexico
}

\begin{abstract}
Introduction: Percutaneous nephrolithotomy is an efficient approach for treatment of different types of kidney stones. Various types of access techniques have been described like sequential dilatation and one-shot procedure. Objective: To determine the differences in time of exposure to $X$-rays and hemoglobin levels between techniques. Methods: Controlled clinical trial. Patients older than 18 years with complex/uncomplicated kidney stones, without urine infection were included. They were assigned randomly to one of the two techniques. Response variables were determined before and $24 \mathrm{~h}$ after procedures. Results: 59 patients were included: 30 underwent one-shot procedure (study-group) and 29 sequential dilatation (control-group). Baseline characteristics were similar. Study group had a lower postoperative hemoglobin decline than control group ( 0.81 vs. $2.03 \mathrm{~g} / \mathrm{dll}$, respectively; $p<0.001)$; $X$-ray exposure time (69.6 vs. $100.62 \mathrm{~s} ; p<0.001$ ) and postoperative creatinine serum levels (0.93 $\pm 0.29 \mathrm{vs}$. $1.13 \pm 0.4 \mathrm{mg} / \mathrm{dl} ; p=0.039)$. No significant differences in postoperative morbidity were found. Conclusion: One-shot technique demonstrated better results compared to sequential dilatation.
\end{abstract}

KEY WORDS: Renal calculi. Nephrolithotomy. One-shot nephrostomy. X-ray exposure time. Hemorrhage.

\section{Introduction}

Kidney stones are a common cause of urological consultation. It is a highly prevalent condition, since estimates indicate that around of $10 \%$ of the population is going to suffer from it sometime in its lifetime ${ }^{1,2}$. This prevalence has doubled over the past 40 years, from a yearly incidence of 54.2 per 100,000 population in 1965 to 114.3 per 100,000 population in 2005. It is more common in men, but the difference between both genders has decreased with time. It has a peak incidence between the fourth and the sixth decades of life. The prevalence increases in arid, warm and dry climates ${ }^{1,3}$.

Percutaneous nephrolithotomy (PNL) is a procedure that consists in creating a tract through the skin and the renal parenchyma in order to reach the collecting system. By means of this access, lithotripsy is performed, or the stones can be extracted using special forceps (with previous nephroscopy). PNL has become the main indication for $1-2-\mathrm{cm}$ staghorn or semi-staghorn calculi ${ }^{4}$. The main minor complications observed in PNL include the need for transfusion (11.2-17.5\%), fever (21-32\%) and urinoma $(0-1 \%)$, whereas major complications can comprise colonic

\section{Correspondence:}

Alejandro González-Ojeda

Avda. Belisario Domínguez, 1000

Col. Independencia

E-mail: avgail5@gmail.com

C.P. 44340 , Guadalajara, Jal., México

Date of reception: 27-09-2016

Date of acceptance: 04-10-2016 avygail5@yahoo.com.mx
DOI://dx.doi.org/10.24875/GMM.M18000055
Gac Med Mex. 2017;153:610-615

Contents available at PubMed www.gacetamedicademexico.com 
(0.2-0.8\%) or pulmonary injury $(0.0-3.1 \%)$, sepsis $(0.3-$ $4.7 \%)$ and death $(0.0-0.3 \%)$. Although patient comorbidity increases the risk of complications, most cases are conservatively resolved or with minimally invasive procedures, with normal postsurgical evolution in $76.7 \%$ of cases $^{1,5-7}$.

Among the PNL approaches, the traditional one consists in progressive dilatation of the tract created to place the access and working sheath. Despite the safety of this procedure, longer time of X-ray (fluoroscopy) exposure has been observed both for the patient and the healthcare personnel, as well as larger blood loss. The one-shot approach would appear to be more traumatic in order for the dimensions required to place the access sheath to be achieved in a single act, but it has been shown to reduce the time of fluoroscopy exposure and the rate of complications ${ }^{7}$. The purpose of this study was to determine if there is benefit deriving from the one-shot procedure in patients with nephrolithiasis in our hospital center.

\section{Methods}

The local committee of health research and ethics of the High Specialty Medical Unit, Specialty Hospital, National Medical Center of the West of the Mexican Institute of Social Security, approved the research protocol with registry number R-2015-1301-107. All patients granted their duly signed informed consent.

This was a single-blind, controlled study carried out at the Urology Department in patients older than 18 years with negative urine culture, positive urine culture without urosepsis, prothrombin activity higher than $80 \%$, with complex uncomplicated lithiasis and stable kidney function, even with replacement therapy. Patients with urosepsis, respiratory function alterations, heart failure and non-controlled renal failure were excluded.

The patients were randomly divided in two groups, by means of choosing a closed envelope, with number 1 being assigned to patients in the control group (CG) and number 2 to patients in the study group (SG).

The time of X-ray exposure during the PNL (timed in seconds) was measured, and variations in hemoglobin values were also determined.

Blood samples were obtained from every patient for complete blood count and creatinine serum concentrations one hour prior to surgery.

The requirements for surgery to be scheduled included having a blood count within normal parameters, and if the patient had renal failure, it was required for him/her to be in adequate conditions as regards cardiac function, respiratory function, etc. All patients were requested a urine culture. Once admitted, prophylactic antibiotic treatment was started (cefalotin, $1 \mathrm{~g}$ intravenously, or ciprofloxacin, $500 \mathrm{mg}$, in case of allergy), which was guided according to the urine culture results. At surgery completion, the patient was taken to the recovery room, where postsurgical care was provided, and once 24 postsurgical hours were completed, blood samples were taken for blood count, creatinine and urea.

Under general anesthesia and with prophylactic antibiotic therapy, the patient was placed in the lithotomy position and cystoscopy with a Storz $21 \mathrm{Fr}$ equipment was used to place a ureter guide and an occlusion ureteric catheter, and $20 \mathrm{~mL}$ of contrast material for fluoroscopic pyelography were administered: Then, $1 \mathrm{~mL}$ air was insufflated into the catheter to place it at the ureteropyelic junction, and the patient was placed in the decubitus prone position for the performance of a puncture with a nephrostomy needle in the calyx selected under fluoroscopic guidance; the extra-rigid guidewire was placed and fascial dilation was performed with an 8-14 Fr polyurethane dilator under fluoroscopic guidance, to then replace it with an Alken guide rod and through it place a $30 \mathrm{Fr}$ caliber Amplatz one-shot dilator to the collecting system. Once the tract was performed, an access sheath was placed, nephroscopy was performed with a Storz 26 Fr nephroscope, the calculus was located and lithotripsy was performed until its fragmentation, to subsequently perform litholapaxy and extraction with grasping forceps or stone basket. A new nephroscopy was carried out, the devices were retrieved and, where indicated, a nephrostomy tube with a two-way $22 \mathrm{Fr}$ caliber Foley catheter was placed; a balloon was insufflated with contrast material and it was localized with fluoroscopy.

Once 24 postsurgical hours were elapsed, samples were obtained for blood count and creatinine and urea determination, and a plain abdominal X-ray was obtained in case the supracostal access was used. At 24-48 postsurgical hours, all patients underwent a descending pyelography in case of having a nephrostomy.

The $\alpha$ error was fixed at 0.05 and $\beta$ error at 0.20 and the $\delta$-value at 11.2 (mean and standard deviation for one-shot fluoroscopy time; $41.2 \pm 9.8$; mean and standard deviation for sequential approach fluoroscopy time: $52.4 \pm 10.3)$. An $n=18$ patients was established in order for each group to be considered adequate, 
according to the mean differences formula for clinical trials based on Dehong et al. ${ }^{8}$ (four randomized clinical trials with a total of 6820 patients) and Li et al. ${ }^{9}$ (four randomized clinical trials with a total of 346 patients) fluoroscopy time.

The descriptive phase was carried out by obtaining raw numbers, percentages and central tendency and dispersion measures (mean \pm standard deviation). The analytical phase was carried out using Fisher's exact test and the chi-square test for qualitative variables. For quantitative variables, Student's t-test or Mann-Whitney U-test were used if the distribution of results was normal or abnormal, respectively. Any $p$-value $<0.05$ was considered statistically significant. The analysis was carried out with the statistical package SPSS for Windows, version 20 (IBM Corp., Armonk, NY, USA).

\section{Results}

The patients were divided in two groups: $E G$, with one-shot technique $(n=30)$, and $C G$, with sequential technique $(n=29)$. The $E G$ was comprised by 15 men and 15 women, with an average age of 49.73 years, and the CG, by 14 men and 15 women, with an average age of 45.44 years. General characteristics and preoperative variables are described in table 1.

The EG had less postoperative decrease in hemoglobin values than the $C G$, with the difference being statistically significant (-0.81 vs. $-2.03 \mathrm{~g} / \mathrm{dL} ; \mathrm{p}<0.001$ ). The same trend was observed for creatinine values, where a decrease in the $E G$ and an increase in the $C G$ were shown, with the difference also being significant $(-0.05$ vs. $+0.19 \mathrm{mg} / \mathrm{dL} ; p<0.001)$. However, no deterioration was observed in renal function. In addition, in the times of exposure to ionizing radiation, significantly shorter times were found in the EG than in the CG (69.6 vs. $100.62 \mathrm{~s} ; \mathrm{p}<0.001)$. The assessed operative variables are detailed in table 2. Days of hospital stay for the EG were, on average, 2.23 days, whereas in the CG they were 2.34 days $(p<0.42)$.

As for complications, one patient of each group required blood transfusion, one patient in the CG had a pleural fistula secondary to a supracostal abscess and no patient had serious hemorrhage, renal hematoma or required other type of intervention. There were no visceral or vascular injuries, or surgical wound infection. Similarly, systemic inflammatory

Table 1. Baseline patient characteristics

\begin{tabular}{|c|c|c|c|c|}
\hline Variable & One-shot technique $(\mathrm{N}=30)$ & Sequential technique $(N=29)$ & $\frac{+}{3}$ & p \\
\hline $\mathrm{BMI}\left(\mathrm{kg} / \mathrm{m}^{2} \pm \mathrm{SD}\right)$ & $28.04 \pm 5.49$ & $27.45 \pm 4.30$ & $\subseteq$ & 0.74 \\
\hline Mean age (years \pm SD) & $49.73 \pm 14.3$ & $45.44 \pm 14.4$ & $\frac{\circ}{0}$ & 0.31 \\
\hline Male gender & 15 & 15 & + & $0.89^{*}$ \\
\hline Female gender & 15 & 14 & 으 & \\
\hline Previous nephrolithotomy & 5 & 6 & ஓ & $0.16^{*}$ \\
\hline Horseshoe kidney & 2 & 3 & $\frac{J}{0}$ & $0.48^{*}$ \\
\hline Calculus size $(\mathrm{cm})$ & $2.23 \pm 1.15$ & $2.52 \pm 1.14$ & $\frac{\circ}{(1)}$ & 0.34 \\
\hline Left kidney & 14 & 16 & $\stackrel{\oplus}{\circ}$ & $0.51^{*}$ \\
\hline Right kidney & 16 & 13 & $\vec{\nabla}$ & \\
\hline Calculus in upper calyces & 3 & 2 & $\frac{\varsigma}{\circ}$ & $0.90^{*}$ \\
\hline Calculus in middle calyces & 15 & 14 & $\vec{\theta}$ & \\
\hline Calculus in lower calyces & 8 & 10 & $\overrightarrow{\bar{\partial}}$ & \\
\hline Staghorn calculus & 4 & 3 & $\frac{n}{c}$ & \\
\hline Hemoglobin (g/dL) & $14.33 \pm 2.08$ & $14.64 \pm 0.96$ & 4 & 0.23 \\
\hline Serum creatinine (mg/dL) & $0.98 \pm 0.42$ & $0.93 \pm 0.37$ & 을 & 0.71 \\
\hline One-tract access & 27 & 24 & ¿ & $0.33^{*}$ \\
\hline Two-tract access & 3 & 5 & & \\
\hline
\end{tabular}


Table 2. Preoperative and postoperative laboratory results, fluoroscopy time and blood transfusion rate

\begin{tabular}{|c|c|c|c|}
\hline Variable & One-shot technique $(\mathrm{N}=30)$ & Sequential technique $(\mathrm{N}=29)$ & p \\
\hline Fluoroscopy exposure time $(s \pm S D)$ & $69.60 \pm 21.38$ & $100.62 \pm 23.54$ & $<0.001^{*}$ \\
\hline Preoperative hemoglobin (g/dL \pm SD) & $14.33 \pm 2.08$ & $14.64 \pm 0.96$ & $0.47^{*}$ \\
\hline Postoperative hemoglobin (g/dL $\pm \mathrm{SD}$ ) & $13.52 \pm 1.79$ & $12.61 \pm 1.78$ & $0.03^{*}$ \\
\hline Pre/post hemoglobin difference ( $\mathrm{g} / \mathrm{dL} \pm \mathrm{SD})$ & $0.81 \pm 0.78$ & $2.03 \pm 1.04$ & $<0.001^{*}$ \\
\hline Preoperative creatinine $(\mathrm{mg} / \mathrm{dL} \pm \mathrm{SD})$ & $0.98 \pm 0.42$ & $0.93 \pm 0.37$ & $0.62^{*}$ \\
\hline Postoperative creatinine (mg/dL $\pm \mathrm{SD}$ ) & $0.93 \pm 0.29$ & $1.13 \pm 0.43$ & $0.039^{*}$ \\
\hline Serum creatinine difference $(\mathrm{mg} / \mathrm{dL} \pm \mathrm{SD})$ & $-0.055 \pm 0.071$ & $+0.19 \pm 0.14$ & $\leq 0.001^{*}$ \\
\hline Blood transfusion rate & $3.44 \%$ & $3.57 \%$ & $0.74^{\dagger}$ \\
\hline
\end{tabular}

${ }^{*}$ Calculated with Student's t-test.

${ }^{\dagger}$ Calculated with Fisher's exact test.

SD: standard deviation.

response, such as signs of tachycardia (6\% of CG patients), fever (6\% of patients in both groups) or leukocytosis (33\% of $E G$ and $31 \%$ of $C G$ patients), as well as the need to be surgically re-intervened (no patient had to), showed no significant differences (Table 3).

According to the Clavien-Dindo classification for surgical complications, which is divided from I to IV according to the severity of complications and the treatment they require, patients were classified as follows: in the $C G$, there were 2 patients with fever, 2 with tachycardia, and 9 with leukocytosis (grade I), one patient who required transfusion (grade II), and one patient with a pleural fistula (grade IIIa); in the $E G$, there were 2 patients with fever, 10 with leukocytosis (grade I), and one who required transfusion (grade II).

\section{Discussion}

PNL has come to change the use of open surgery for a minimally invasive procedure, thus reducing complications for the patient, as well as days of hospital stay ${ }^{10}$. Even with the advances in surgical technique, patients and healthcare personnel still have to spend prolonged surgical periods and time of radiation exposure.

The balloon dilation technique is the best accepted method for the percutaneous surgery tract to be performed, but its elevated cost limits its systematic use; however, the study conducted by Yamaguchi et al. ${ }^{7}$, associates balloon dilation with longer surgical time, which entails more bleeding. Amplatz or metallic dilator dilation increases the time of radiation exposure and consumes more trans-operative time. The reason
Table 3. Postoperative mortality and morbidity

\begin{tabular}{|c|c|c|c|c|}
\hline Variable & $\begin{array}{l}\text { One-shot } \\
\text { technique } \\
(\mathrm{N}=30)\end{array}$ & $\begin{array}{c}\text { Sequential } \\
\text { technique } \\
(\mathrm{N}=29)\end{array}$ & & $\mathbf{p}$ \\
\hline Hospital stay (days) & $2.23 \pm 0.56$ & \multicolumn{2}{|c|}{$2.34 \pm 0.48$} & $0.42^{*}$ \\
\hline \multicolumn{5}{|l|}{ Nephrostomy } \\
\hline Yes & 24 & 25 & 은 & $0.52^{\dagger}$ \\
\hline No & 6 & 4 & $\frac{2}{0}$ & \\
\hline \multicolumn{5}{|l|}{ Fever } \\
\hline Yes & 2 & 2 & $\frac{5}{3}$ & $0.51^{\ddagger}$ \\
\hline No & 28 & 27 & $\mp$ & \\
\hline \multicolumn{5}{|l|}{ Leukocytosis } \\
\hline Yes & 10 & 9 & $\subseteq$ & $0.85^{\dagger}$ \\
\hline No & 20 & 20 & 층 & \\
\hline \multicolumn{5}{|l|}{ Tachycardia } \\
\hline Yes & 0 & 2 & 을 & $0.23^{\ddagger}$ \\
\hline No & 30 & 27 & 은 & \\
\hline \multicolumn{5}{|l|}{ Transfusion } \\
\hline Yes & 1 & 1 & $\underline{\cup}$ & $0.74^{\ddagger}$ \\
\hline No & 29 & 28 & $\frac{0}{2}$ & \\
\hline \multicolumn{5}{|c|}{ Injure to neighboring organs } \\
\hline Yes & 0 & 2 & - & $0.23^{\ddagger}$ \\
\hline No & 30 & 28 & 을 & \\
\hline \multicolumn{5}{|l|}{ Complications } \\
\hline Yes & 1 & 1 & $\subseteq$ & $0.74^{\ddagger}$ \\
\hline No & 29 & 28 & $\underline{\bar{\sigma}}$ & \\
\hline
\end{tabular}

${ }^{*}$ Calculated with Student's t-test.

${ }^{+}$Calculated with the Chi-square test.

₹Calculated with Fisher's exact test.

why the one-shot technique has increased its acceptance among urologists is its low cost, as well as reduced surgical time and fluoroscopy duration ${ }^{7-9}$.

Frattini et al. ${ }^{11}$ conducted a study with 78 patients where they compared sequential dilation, the one-shot technique and balloon dilation as regards X-ray exposure, blood loss and cost-benefit. Their results 
indicate that the one-shot technique is comparable to the other two dilation techniques, since it didn't produce a morbidity increase and significantly reduced radiation exposure and costs and, in addition, had a lower rate of hemorrhage in comparison with both the other techniques. In our study, the costs of materials were not assessed, but this could be an important variable in favor of the one-shot technique, since only a single disposable dilator, inserted in a reusable Alken guide, is required, which further reduces costs.

A highly important complication associated with percutaneous renal surgery is hemorrhage. In the study by Stoller et al. ${ }^{12}$, factors associated with blood loss in PNL were assessed in 127 patients, with no increase being found in the risk of hemorrhage using fascial dilators ${ }^{12}$. In our study, fascial dilation was used with 8-14 Fr calibers in all cases, without this being associated with hemorrhage of the tract. In addition, this type of dilation can improve access in patients with previous surgery.

Several studies have directly compared the oneshot technique with sequential dilation using Amplatz or metallic dilators. One-shot dilation has been shown to have lower blood transfusion requirements ${ }^{5,6,10}$. Our study evaluated blood loss with hemoglobin serum values determination prior and after the intervention, with a significant decrease being found in the patients who underwent one-shot dilation versus sequential dilation ( $p<0.001$ ). Although these results are statistically significant, no difference was found in the number of blood transfusions between both groups $(p=0.74)$.

Aminsharifi et al..$^{13}$ conducted a study that revealed the harm caused to the renal parenchyma by comparing one-shot dilation against sequential dilation through technetium 99 uptake in a dimercaptosuccinic acid (DMSA) scintigraphy, and found that 14 of 29 patients with the one-shot technique had a new parenchymal scar, in comparison with 2 of 29 patients in the sequential dilation group. In our study, renal function was tried to be indirectly measured with creatinine serum values; however, both technetium 99 uptake in DMSA scintigraphy and creatinine serum values are not the diagnostic modality of choice to determine the renal function. Better studies are required in order to determine short and long-term renal damage; however, our findings in creatinine serum values suggest that the one-shot technique is safer in terms of renal function preservation, but not for decreasing renal scarring. In spite of having obtained statistically significant results with regard to hemoglobin and creatinine values, they are not regarded as clinically significant because in no group was there renal damage or renal function deterioration.

Frattini et al. ${ }^{11}$ refer renal hypermotility and previous surgery as relative contraindications for the one-shot technique because they increase the rate of associated complications, such as vascular or adjacent structures' damage. When any of these risk factors is identified, performing the nephrostomy tract with conventional technique is recommended ${ }^{11}$. In order to reduce these complications, the applied radial force is crucial, using rotational movements during the gradual process to this way reduce the displacement of the renal unit and the failure rate to $7 \%$ in patients with previous surgery $8,13,14$.

Among the limitations when performing this study, we found the difficulties to directly assess the renal function. Although the surgeon's experience influences on the outcome of every surgery, we do not consider the fact that the procedures performed for this study were carried out by a single surgeon to be a limitation.

In our study, 5 patients in the EG and 6 in the CG were found to have had previous surgery, but performing the surgery with no difficulty was possible in all of them, and it was not associated with an increase in the hemorrhage rate or damage to neighboring structures. Only one patient of the EG had renal hypermotility during the procedure, making it impossible for the oneshot technique to be performed, which was associated with fluoroscopy time increase, and the dilation had to be sequentially completed, without further complications. Therefore, sequential dilation is recommended in those cases where renal motility is detected.

An important finding in this study was that the surgeon who performed the procedures noted that the type of dilation affected the visual field during surgery. This was associated with trans-surgical hemorrhage, which was higher in the CG. However, this variable was not measurable. Further studies should be conducted on this particular subject, owing to the limited literature on the problem, as well as studies on the cost-effectiveness of this technique, especially with regard to the benefit and specifically-measured renal function preservation.

\section{Conclusions}

The one-shot technique is safer and more effective for performing the nephrostomy tract in patients with nephrolithiasis regardless of size, location, history and 
anatomic variation, without complication rates being increased and considerably reducing radiation times and the amount of hemorrhage.

The use of the one-shot technique is recommended in patients with nephrolithiasis who are candidates for PNL owing to the benefits it confers both to the patient and the healthcare personnel.

\section{Conflicts of interests}

The authors declare there are no conflicts of interests. All the authors approved the manuscript final version.

\section{Funding}

No funding was required.

\section{References}

1. Wein K. Litiasis urinaria: etiología, diagnóstico y manejo médico. En: Wein A, Kavoussi L, Novick A, et al., editores. Campbell-Walsh. Urología. 10. ${ }^{a}$ ed. Philadelphia: Saunders Elsiever; 2010. p. 1257-410.

2. Middela S, Papadopoulos G, Srirangam S, et al. Extracorporeal shock wave lithotripsy for ureteral stones: do decompression tubes matter? Urology. 2010;76:821-5.
3. Türk C, Knoll T, Köhrmann KU. New guidelines for urinary stone treatment. Controversy or development? Urologe A. 2008;47:591-3.

4. Wen CC, Nakada SY. Treatment selection and outcomes: renal calculi. Urol Clin North Am. 2007;34:409-19.

5. Castillo O, Pinto I, Díaz M, et al. Cirugía percutánea de litiasis coraliforme. Rev Chil Cirug. 2008;60:393-7.

6. Michel MS, Trojan L, Rassweiler JJ. Complications in percutaneous nephrolitotomy. Eur Urol. 2007;51:899-906.

7. Yamaguchi A, Skolarikos A, Buchholz NP, et al. Clinical Research Office of the Endourological Society Percutaneous Nephrolithotomy Study Group. Operating times and bleeding complications in percutaneous nephrolithotomy: a comparison of tract dilation methods in 5,537 patients in the Clinical Research Office of the Endourological Society Percutaneous Nephrolithotomy Global Study. J Endourol. 2011;25:933-9.

8. Dehong $C$, Liangren $L$, Huawei $L$, et al. A comparison among four tract dilation methods of percutaneous nephrolithotomy: a systematic review and meta-analysis. Urolithiasis. 2013;41:523-30.

9. Li Y, Yang L, Xu P, et al. One-shot versus gradual dilation technique for tract creation in percutaneous nephrolithotomy: a systematic review and meta-analysis. Urolithiasis. 2013;41:443-8.

10. Deem S, DeFade B, Modak A, et al. Percutaneous nephrolithotomy versus extracorporeal shock wave lithotripsy for moderate size kidney stones. Urology. 2011;78:739-43.

11. Frattini A, Barbieri A, Salsi $P$, et al. One-shot: a novel method to dilate the nephrostomy access for percutaneous lithotripsy. J Endourol. 2001; 15:91923.

12. Stoller ML, Wolf JS Jr, St Lezin MA. Estimated blood loss and transfusion rates associated with percutaneous nephrolithotomy. J Urol. 1994; 152:1977-81.

13. Aminsharifi A, Alavi M, Sadeghi G, et al. Renal parenchymal damage after percutaneous nephrolithotomy with one-stage tract dilation technique: a randomized clinical trial. J Endourol. 2011;25:927-31.

14. Amjadi M, Zolfaghari A, Elahian A, et al. Percutaneous nephrolithotomy in patients with previous open nephrolithotomy: one-shot versus telescopic technique for tract dilatation. J Endourol. 2008;22:423-5. 